\title{
EQUOTERAPIA COMO MÉTODO DE TRATAMENTO FISIOTERAPÊUTICO
}

\author{
João Antônio Simeoni Romagnoli \\ Mestrando em Ciências do Movimento Humano (UDESC) \\ ja_romagnole@hotmail.com
}

\author{
Daniel Vicentini de Oliveira \\ Doutorando em Gerontologia (UNICAMP) \\ d.vicentini@hotmail.com
}

\section{Mateus Dias Antunes}

Mestrando em Promoção de Saúde (UNICESUMAR)

mateus_antunes03@hotmail.com

\author{
José Roberto Andrade do Nascimento Junior \\ Doutor em Educação Física (UEM) \\ jroberto.jrs01@gmail.com
}

\author{
Emília Maria Barbosa Carvalho Kempinski \\ Mestre em Saúde Coletiva (UERJ) \\ emiliakempinski@gmail.com
}

\section{RESUMO}

A equoterapia é um método de tratamento que utiliza o cavalo dentro de uma abordagem interdisciplinar, nas áreas de saúde, educação e equitação, buscando o desenvolvimento biopsicossocial de pessoas com deficiência e/ou com necessidades especiais, o objetivo deste trabalho foi traçar o perfil dos alunos atendido pelo centro de equoterapia visando proporcionar uma quantificação dos atendimentos aos pacientes, a fim de direcionar os estudos de forma a abranger todas as enfermidades encontradas e também para conhecer mais sobre a equoterapia e suas técnicas. Esta pesquisa se caracteriza em um estudo observacional, descritivo e retrospectivo. Participaram 20 alunos, 9 do sexo masculino e 11 do sexo feminino. Os resultados evidenciaram que o público mais atendido por essa terapia, foram crianças portadoras da paralisa cerebral que na sua maioria necessitam de um tratamento interdisciplinar devido seu distúrbio motor evidente. Conclui-se que a equoterapia é um método terapêutico em evolução e pouco explorado, porém o conhecimento dessas variáveis visa auxiliar uma abordagem diferenciada diante das diversas situações encontradas, sendo necessária uma abordagem interdisciplinar.

Palavras-chave: Equoterapia; Fisioterapia; Terapia com o uso do cavalo.

\begin{abstract}
The equine therapy is a treatment method that uses the horse within an interdisciplinary approach in the areas of health, education and riding, seeking the biopsychosocial development of people with disabilities and / or special needs, the aim of this study was to trace the profile of students attended the hippotherapy center aiming to provide a quantification of care to patients, in order to direct the studies to cover all diseases found and also to learn more about the hippotherapy and techniques. This research is characterized in an observational, descriptive and retrospective study. 20 students participated, 9 males and 11 females. The results showed that the public more entertained by this therapy, were children with cerebral paralyzes which mostly require an interdisciplinary treatment because their disorder evident engine. We conclude that equine therapy is a therapeutic method in evolution and little explored, but the knowledge of these variables aims to
\end{abstract}


assist a differentiated approach on the various situations encountered, requiring an interdisciplinary approach.

Keywords: Hippotherapy; Physical Therapy; Therapy with the use of the horse.

\section{INTRODUÇÃO}

É de grande importância o estudo das patologias que provocam alterações neurofuncionais nos indivíduos. Sabe-se que, mesmo com o grande desenvolvimento da medicina, pouco se demonstra na atualidade em casos de malformações, síndromes e paralisia cerebral, entre outros, sendo a equoterapia nestes casos um recurso importante para promover avanços no desenvolvimento neuropsicomotor desses indivíduos (CHAMPAGNE; CORRIVEAU; DUGAS, 2016).

A equoterapia é um método de tratamento que utiliza uma abordagem interdisciplinar por meio do cavalo, nas áreas de saúde, educação e equitação, buscando o desenvolvimento global de pessoas com deficiência e/ou com necessidades especiais, O Uso do cavalo ocorre devido ser um animal dócil, de porte e força, que se deixa manusear e montar. Dessa forma, o cavalo e o praticante criam um relacionamento afetivo importante, estabelecendo uma relação harmoniosa e atuação mútua (BECHEVA et al., 2016)

A reabilitação física e mental de pessoas portadoras de necessidades especiais, com dificuldades motoras e deficiências físicas, mentais e/ou psicológica é promovida por meio da equoterapia. O cavalo, neste método, entra como um agente facilitador, proporcionando aos praticantes ganhos tanto no aspecto físico como no psicológico, exigindo um trabalho muscular intenso e contribuição para adequação do tônus, melhora da coordenação e do equilíbrio (JANG et al., 2016).

A equoterapia é uma alternativa que visa melhorar a qualidade de vida dos pacientes, que, em muitos casos não caminham ou passam um longo período sentado a equoterapia possibilita trabalhar o equilíbrio, lateralidade, a psicomotricidade fina e grossa, e outras habilidades relacionadas à educação. Quando a criança começa a se equilíbrar torna-se apta a ver o mundo em posição vertical, a sustentação da cabeça é de suma importância para que a criança possa visualizar os diversos estímulos e tudo aquilo que lhe é ensinado (HSIEH et al., 2016)

Neste método terapêutico podemos trabalhar o cognitivo do paciente estimulando a sensibilidade tátil, visual, auditiva e olfativa pelo ambiente e pelo uso do cavalo promovendo assim a organização e a consciência corporal e aumentando a auto- estima, facilitando a integração social, motivando o aprendizado, encorajando o uso da linguagem, ensinando a importância de regras e disciplinas e aumentando a capacidade de decisões e independência em diferentes situações (SANTOS, 2005).

O cavalo realiza movimentos e os transmite ao cavaleiro, gerando um mecanismo de resposta. Embora os movimentos se processarem de maneira rápida, eles não chegam a serem tão rápidos a fim de impedir seu entendimento pelo cérebro dao indivíduo. A repetição, ritmo, simetria e cadência fazem com que suas repostas surjam muito rapidamente, destacando como uma das principais vantagens da utilização do cavalo (SOUZA; SILVA, 2015).

Assim, o animal oferece uma vasta diversidade de movimentos quando o indivíduo está sbre o seu dorso por meio de três andaduras, o passo, o trote e o galope. Esses movimentos específicos favorecem inclusive o passo, devido a regularidade, pois o ritmo proporciona um ritmo que não produz impacto em quem monta (LONG, 2014).

As principais indicações são para sequelas de lesões medulares e cerebrais, poliomielite, plegias, sequelas ortopédicas, cardiovasculares e respiratórias, disfunções motoras e de coordenação, bem como, distúrbios comportamentais sociais, sensoriais e mentais (KWON et al., 2015).

Durante a equoterapia o fisioterapeuta tem a função de facilitar e conduzir os movimentos normais e inibir padõres anormais durante a sessão (SMÍSKOVÁ, 2014). Junto ao instrutor de equitação, o fisioterapeuta avalia as condições de trabalho, observando se o praticante tem participado durante da sessão do treinamento e se o animal está vindo ao trabalho descontraído para que evite situações impróprias durante 
a terapia (LEE; KIM; NA, 2014).

A equoterapia tem como objetivo neuromotores globais: ajuste tônico, equililbrio, alinhamento corporal, coordenação motora, fortalecimento e resistência muscular (MEDEIROS, 2008). De acordo com sua patologia, precauções e quadro clínico a equoterapia pode intervir devido ao uso de vários materiais que são benéficos ao tratamento fisioterápico, utilizando assim, de uma avaliação ergonômica, para obter melhores formas de segurança no seu atendimento, onde serão alcançados seus objetivos (THOMPSON; KETCHAM; HALL, 2014). A união da fisioterapia e a equoterapia em tratamento com pacientes com paralisia cerebral propiciou aumento na força de membros inferiores (MMII) e equilíbrio resultando na melhoria da marcha, além do desenvolvimento da integração social (LEE; PARK; KIM, 2015).

Dessa forma a equoterapia é uma terapia em evolução e pouco explorada, apesar de existir a alguns anos, isso acontece devido aos poucos estudos que se tem sobre esta área de tratamento. O objetivo deste estudo foi traçar o perfil dos alunos atendido pelo centro de equoterapia visando proporcionar uma quantificação dos atendimentos aos pacientes, a fim de direcionar os estudos de forma a abranger todas as enfermidades encontradas e também para conhecer mais sobre a equoterapia e suas técnicas.

\section{METODOLOGIA}

Trata-se de um estudo observacional, descritivo e retrospectivo. Os critérios de inclusão para este estudo foram indivíduos com idade entre 0 a 18 anos, com diagnóstico de distúrbios neurofuncionais e estarem matriculados no Centro de Equoterapia na Cidade de Maringá-PR.

O levantamento dos dados ocorreu por meio de entrevistas realizadas com os pais dos alunos buscando informações sobre sexo, idade e diagnóstico clínico para caracterização do perfil. Foi realizado o acompanhamento das sessões de equoterapia e descrição do trabalho realizado com cada aluno, este acompanhamento se repetiu por 3 sessões consecutives para: descrever o atendimento para relacionar com o perfil de cada criança e analisar todos os dados coletados para a elaboração da discussão.

Inicialmente o estudo foi aprovado pelo Comitê de Ética da Faculdade Ingá pela resolução no $466 / 12$ e parecer $n^{\circ}$ 0244/11. Para a coleta de dados, os pesquisadores entraram em contato com os responsáveis pelo Centro de Equoterapia de Maringá-PR, com o intuito de esclarecer os objetivos e os procedimentos que seriam utilizados durante a pesquisa. A seguir, foram agendadas datas para a assinatura do Termo de Consentimento Livre e Esclarecido pelos pais.

Os dados foram analisados no software SPSS versão 22.0. Foi utilizada a distribuição de freqüência (absoluta e relativa) para a caracterização dos resultados.

\section{RESULTADOS}

Foram entrevistados pais de 20 crianças que frequentavam o centro de equoterapia. Destes 20 alunos que utilizam o centro de equoterapia, $55 \%$ eram do sexo masculino e $45 \%$ do sexo feminino. Em relação ao diagnóstico clínico, os dados coletados evidenciaram que há uma grande prevalência da paralisa cerebral, sendo que $60 \%$ dos alunos apresentavam este diagnóstico, $15 \%$ apresentam diagnóstico com ADNPM que é o termo usado quando o paciente não possui diagnóstico fechado, $25 \%$ apresentaram diagnóstico de autismo,síndromes metabólicas e outras patologias, as quais na tabela se encontram como diversos. Em relação à idade dos alunos, $40 \%$ tem idade entre 3 e 5 anos, $40 \%$ tem idade entre 6 e 10 anos, $10 \%$ idade entre 11 a 14 anos e $10 \%$ idade entre 15 a 18 anos, não foi encontrado nenhum aluno com idade de 0 a 2 anos,este dado sugere que a criança apresente no mínimo a aquisição motora de tônus postural integro e total para realizar a terapia (Tabela 1$)$. 
Tabela 1. Perfil das crianças no Centro de Equoterapia a cidade de Maringá - PR.

\begin{tabular}{lcc}
\hline VARIÁVEIS & N & \% \\
\hline Sexo & 11 & 55 \\
Masculino & 9 & 45 \\
Feminino & & \\
Diagnóstico & 12 & 60 \\
Paralisica Cerebal & 3 & 15 \\
ADNPM & 5 & 25 \\
Diversos & & \\
Idade & & - \\
$0-2$ anos & - & 40 \\
$3-5$ anos & 8 & 40 \\
6-10 anos & 8 & 2 \\
$11-14$ anos & 2 & 2 \\
$15-18$ anos & 2 & \\
Frequência dos alunos & 25 \\
1 vez na semana & 25 \\
2 vezes na semana & 15 & \\
\hline
\end{tabular}

Legenda: ADNPM: paciente não possui diagnóstico fechado

Pode-se observar na Tabela 2 em relação ao tratamento em equipe interdisciplinar os mais realizados foram a fisioterapia solo e a hidroterapia, ambas com $30 \%$.

Tabela 2. Frequência de tratamento interdisciplinar das crianças do Centro de Equoterapia a cidade de Maringá - PR.

\begin{tabular}{lcc}
\hline VARIÁVEIS & N & \% \\
\hline Tipos & & \\
Fisioterapia Solo & 6 & 30 \\
Fonoaudiologia & 6 & 30 \\
Hidroterapia & 3 & 15 \\
Psicologia & 1 & 5 \\
Terapia Ocupacional & 1 & 5 \\
Outros & 3 & 15 \\
\hline
\end{tabular}

A montaria mais utilizada durante a terapia foi a montaria individual com $80 \%$ das crianças, onde o terapeuta fica do lado do cavalo incentivando e mostrando como deve ser realizado os exercícios. Em relação as atividades executadas na sessão, os exercícios de fortalecimento foram realizados com maior frequência. Os resultados estão descritos nas tabela 3. 
Tabela 3. Frequência do tipo de montaria e de atividades realizadas nas crianças do Centro de Equoterapia a cidade de Maringá - PR.

\begin{tabular}{lcc}
\hline VARIÁVEIS & N & \% \\
\hline Tipos de montaria & & \\
Montaria individual & 16 & 80 \\
Montaria dupla & 4 & 20 \\
Atividades realizadas & & \\
Alongamento & 6 & 30 \\
Atividades lúdicas & 11 & 55 \\
Fortalecimento & 12 & 60 \\
\hline
\end{tabular}

Legenda: Algumas crianças realizavam mais de uma atividade durante a sessão de equoterapia.

\section{DISCUSSÃO}

O objetivo deste estudo foi traçar o perfil dos alunos atendido pelo centro de equoterapia visando proporcionar uma quantificação dos atendimentos aos pacientes, a fim de direcionar os estudos de forma a abranger todas as enfermidades encontradas e também para conhecer mais sobre a equoterapia e suas técnicas. O mesmo mostra um dado importante, no qual a maioria dos alunos possuíam idade de 3 a 10 anos. Alguns autores relatam que quanto mais precoce a realização do diagnóstico e o tratamento de afecções neurológicas, mais eficaz será o resultado, bem como é de suma importância avaliar a criança de forma global e integrada para um diagnóstico e tratamento correto e precoce (TORQUATO et al., 2013; BACCARIN, MOTA, 2013; JUSTI; GRUBITS, 2014).

O cavalo incluso nas terapias pode ser considerado como um conjunto de técnicas reeducativas, que atuam para superar dados motores, sensoriais, cognitivos e comportamentais, por meio de uma atividade lúdica despostiva, proporcionando todas as condições escolhidas como importantes ao tratamento destes paciente (SILKWOOD-SHERER et al., 2012).

Em relação à frequência dos alunos na equoterapia $75 \%$ realizavam uma vez na semana, e $25 \%$ duas vezes na semana. Isto se deve, devido ao fato da fisioterapia com o cavalo ter um custo relativamente alto, desta forma muitas familias carentes não podem pagar por esse tratamento. E também pelo fato de que muitas pessoas cadeirantes dependem do transporte público para chegar ao centro, encontrando grandes dificuldades no acesso (BARBOSA; MUNSTER, 2014; KOCA; ATASEVEN, 2015).

O tratamento interdisciplinar realizado em conjunto com a equoterapia é de grande importância, pois a maioria dos individuos que frequentavam o centro não posssuiam apenas um distúrbio, e sim vários associados necessitando desse tipo de tratamento (MORAES et al., 2015). A grande proporção de crianças que realizam fisioterapia solo e fonoaudilogia pode ter ocorrido devido ao fato de que a paralisia cerebral gera um distúrbio motor evidente necessitando desses tratamentos (FLORES et al., 2014). Esta abordagem interdisciplinar, também é justificada devido esse método terapêutico e educacional utilizando o cavalo nas áreas da saúde, educação e equitação, a fim de buscar o desenvolvimento biopsicossocial de pessoas com necessidades especiais.

Equoterapia é aplicada por uma equipe interdisciplninar especializada composta por fisioterapeutas, instrutor de euitação, auxiliar lateral e guia e veterinário, bem como podendo ser completada por terapeutas ocupacionais, psicólogis, pedagosos e fonoaudiologos, para uma boa interação entre a equipe e obtenção de resultados benéficos (BARRETO et al., 2007).

Um estudo realizado com 20 crianças de 13 anos com transtorno de déficit de atenção hipertatividade (TDAH) sobre o uso da equoterapia, revelou que é eficaz na melhora dos sintormas gerados por esse déficit 
(BYONGSU et al., 2015). Esse dado vai de encontro com os resultados deste estudo que além de alunos com o diagnostico de paralisia cerebral existem diagnosticos de TDAH que também realizam esse tratamento.

Darnay e Zamora (2015) sugerem que a equoterapia pode beneficiar crianças com distúrbios neurofuncionais, pelo fato de induzir a uma resposta autonômica agura durante a execução da terapia. Os profissionais que utilizam o cavalo como instrumento terpêutico precisam ter o conhecimento da biomecênica do animal, o seu comportamento, bem como seus sitemas de comunicação (SILVA; GRUBITS, 2004).

Segundo Medeiros \& Dias, (2002) a montaria representa a fase central da sessão, em que o inicio das atividades propostas inicial com o indivíduo sobre o dorso anima. A montaria dupla é realizada quando o praticante não tem condições físicas e/ou mentais para manter-se cozinho sobre o animal. Sendo assim, necerrário um terapeuta para montar o cavalo, danto o apoio (LERMONTOV, 2004). No presente estudo apenas $20 \%$ das crianças necessitava desse tipo de montaria.

Nessa fase o programa é necessariamente da área de reabilitação, voltada para as pessoas portadoras de deficiência física e/ou mental onde o cavalo atua, como um agente cinesioterapêutico (LIPORONI; OLIVEIRA, 2010). Em relação à montaria individual ou em dupla, ela é realizada de acordo com o quadro do indivíduo e o objetivo proposto, no qual os principais são a aquisição do controle cervical por meio do movimento do cavalo, e a partir daí, evolui para a manutenção do controle postural do tronco (ESPINDULA et al., 2014).

Contudo, além dos movimentos de ajustes posturais exigidos, como alternância de movimentos com os braços e dissocições de cinturas para que o indivíduo fique montado sobre o cavalo, após aquisição desse controle, a coordenação motora grossa e fina é estimulada por meio de diversas maneiras como o toque no animal com movimentos espcíficos, uso de objetos, ou até mesmo escovando o cavalo (SCHELBAUER; PEREIRA, 2012).

O relaxamento é comumente indicado para o início dos trabalhos de correção postural após a conscientização corporal que ele proporciona. Ele deve ser buscado em grande parte das situações na equoterapia com segurança e conforto. Há alguns meios para se facilitar que o praticante consiga essa situação. Ele pode ser realizado em decúbito ventral ou dorsal estando sobre a garupa do cavalo, sempre em ambiente calmo (SMÍSKOVÁ, 2014).

Mesmo que a fisioterapia convencional tem se mostrado eficiente na reabilitação de pacientes neurológios na melhora do equilíbrio e força, as crianças que realizam equoterapia apresentam uma maior interação entre o ambiente, o animal e o terapeuta adquirindo uma melhor sociabilização. Já nas sessões de fisioterapia convencional, geralmente o tratamento é realizado individualmente, e o contato é apenas entre o paciente e p terapeuta (TORQUATO et al., 2013).

O cavalo é utilizado como um instrumento cinesioterapêutico que melhora o alinhamento corporal, controle das sinergias globais e aumento do equilíbrio estático, bem como o dinâmco. No caminhar do cavalo por trinta minutos de terapia, o paciente executa de 1.800 a 2.250 ajustes tônicos capazes de atuar no sistema nervoso central (GALVÃO et al., 2010).

A equoterapia faz com o que portador de necessidades especiais menos dependente, pois, traz benefícios para a mente e para o corpo, melhora o equilíbrio dinâmico e estático e aprimora a coordenação motora (CABIDDU et al., 2016). Um aspecto muito importante é de que o tratamento com os pacientes neurológicos, a maio parte de sua reabilitação depende de sua própria motivação em relação à proposta terapêutica que lhe será ofertada (GIAGAZOGLOU et al., 2012).

Já está comprovado que a equoterapia é uma proposta alternativa eficaz, uma vez que auxilia na aquisição de padrões esses ciais do desenvolvimento, preparando o paciente para uma subsequente atividade motora mais complexa, aumentando a sua socialização dando condições para que possam simultaneamente desenvolver outras habilidades que estão relacionas internamente com o desenvolvimento da capacidade global motora (LIPORONI; OLIVEIRA, 2010). 
A Comissão de Seguridade Social e Família aprovou o Projeto de Lei 5499/05, do Senado Federal, que inclui a equoterapia entre os serviços especializados oferecidos pelo Sistema Único de Saúde. Fica evidente, portanto, que o método é baseado cientificamente em pesquisas que justifica os benefícios desta abordagem terapêutica. Faz-se necessário as práticas que estimulem a equoterapia como um instrumento terapêutico pelo Sistema Único de Saúde proporcionando um importante avanço na busca da integração e reabilitação da pessoa portadora de necessidades especiais (ARAÚJO; RIBEIRO; SILVA, 2010).

\section{CONCLUSÕES}

Com o presente estudo foi possível conhecer o perfil, bem como verificar outras variáveis relacionada ao tratamento das crianças. Destaca-se que a maior prevalência foi do sexo masculino, e que o tipo de alteração neurológica foi de paralisia cerebral. Desta forma, o conhecimento dessas variáveis visa auxiliar uma abordagem diferenciada diante das diversas situações encontradas, sendo necessário uma abordagem interdisciplinar. Assim, o ambiente da equoterapia pode ser explorado pelo fisioterapêuta como uma alternativa de reabilitação para diferentes patologiase graus de acometimento, pois o cavalo atua como um instrumento cinecioterapêutico no atendimento das crianças com necessidades especiais a fim de promover uma melhora motora do alinhamento corporal, para o controle das sinergias globaise aumento do equilíbrio, além de promover a prática lúdicas por meio de brincadeiras e a socialização dessas crianças.

\section{REFERÊNCIAS}

ARAÚJO, A. E. R. A.; RIBEIRO, V. S.; SILVA, B. T. F. A equoterapia no tratamento de crianças com paralisia cerebral no Nordeste do Brasil. Fisioter. Bras, v. 11, n. 1, p. 4-8, 2010.

BACCARIN, R. Y. A.; MOTA, C. C. A equoterapia é um método educacional e terapêutico. Boletim APAMVET, v. 4, n. 2, p. 6-7, 2013.

BARBOSA, G. O.; MUNSTER, M. A. V. O efeito de um programa de equoterapia no desenvolvimento psicomotor de crianças com indicativos de transtorno de déficit de atenção e hiperatividade. Revista Brasileira de Educação Especial., v. 20, n. 1, p. 69-84, 2014.

BARRETO, F.; GOMES, G.; SILVA, I. A. S.; GOMES, A. L. M. Proposta de um programa multidisciplinar para portador de Síndrome de Down, através de atividades da equoterapia, a partir dos princípios da motricidade humana. Fitness \& Performance Journal, v. 6, n. 2, p. 82-88, 2007.

BECHEVA, M.; GEORGIEV, D.; OBRESHKOVA, D.; PETKOVA, V. Hippotherapy: integrated approach in children with cerebral palsy (CP). World Journal of Pharmacy and Pharmaceutical Sciences., v. 5, n. 7, p. 9-17, 2016.

BYONGSU, J.; SONG, J.; KIM, S.; LEE, J.; SHIN, H. Y.; KWON, J. Y.; KIM, Y. H.; JOUNG, Y. S. Equine-assisted activities and therapy for treating children with attention-deficit/hyperactivity disorder. The Journal of Alternative and Complementary Medicine, v. 21, n. 9, p. 546-553, 2015.

CABIDDU, R.; BORGHI-SILVA, A.; TRIMER, R.; TRIMER, V.; RICCI, P. A., MONTEIRO, C. I. et al. Hippotherapy acute impact on heart rate variability non-linear dynamics in neurological disorders. Physiology \& behavior, v. 159, n. 1, p. 88-94, 2016.

CHAMPAGNE, D.; CORRIVEAU, H.; DUGAS, C. Effect of hippotherapy on motor proficiency and function in children with cerebral palsy who walk. Physical \& Occupational Therapy in Pediatrics., v. 8, $\mathrm{n}$. 28, p. 1-13, 2016.

DARNAY, S; ZAMORA, A. M. P. L. La hipoterapia como complemento terapéutico en diplejía "parálisis cerebral". Cienc. Salud., v. 5, n. 2, p. 30-37, 2015.

ESPINDULA, A. P.; ASSIS, A., SALOMÃO, I.; SIMÕES, M.; RIBEIRO, M. F.; FERREIRA, A. A.; FERRAZ, P. F.; CARDOSO, I. C.; EMANUEL JÚNIOR, D.; TEIXEIRA, V. P. A. Material de montaria 
para equoterapia em indivíduos com síndrome de Down: estudo eletromiográfico. ConScientiae Saúde, v. 13, n. 3, p. 349-56, 2014.

FLORES, F. M.; SOUSA, L. S.; MENEZES, K. M.; COPETTI, F.; TREVISAN, C. M. Quality of life in multiple sclerosis patients participating in therapeutic horseback riding. ConScientiae Saúde, v. 13, n. 1, p. 39-46, 2014.

GAlVÃO, A.; SUTANI, J.; PIRES, M. A.; PRADA, S. H. F.; CORDEIRO, T. L. Estudo de caso: A equoterapia no tratamento de um paciente adulto portador de ataxia cerebelar. Rev Neurocienc, v. 18, n. 3, p. 353-358, 2010.

GIAGAZOGlOU, P.; ARABATZI, F.; DIPLA, K.; LIGA, M.; KELLIS, E. Effect of a hippotherapy intervention program on static balance and strength in adolescents with intellectual disabilities. Research in developmental disabilities, v. 33, n. 6, p. 2265-2270, 2012.

HSIEH, Y. L.; YANG, C. C.; SUN, S. H.; WANG, T. H.; LUO, H. J. Effects of hippotherapy on body functions, activities and participation in children with cerebral palsy based on ICF-CY assessments. Disability and Rehabilitation., p. 1-11, 2016.

JANG, C. H.; JOO, M. C.; NOH, S. E.; LEE, S. Y.; LEE, D. B.; LEE, S. H.; KIM, H. K.; PARK, H. I. Effects of Hippotherapy on Psychosocial Aspects in Children With Cerebral Palsy and Their Caregivers: A Pilot Study. Annals of rehabilitation medicine., v. 40, n. 2, p. 230-6, 2016.

JUSTI, J.; GRUBITS, H. B. Equoterapia e reabilitação em saúde. Revista Interdisciplinar de Estudos em Saúde, v. 3, n. 2, p. 42-54, 2014.

KOCA, T. T.; ATASEVEN, H. What is hippotherapy? The indications and effectiveness of hippotherapy. North Clin Istanbul.,v, 2, n. 3, p. 247-52, 2015.

KWON, J. Y.; CHANG, H. J.; LEE, J. Y.; HA, Y.; LEE, P. K.; KIM, Y. H. Effects of hippotherapy on gait parameters in children with bilateral spastic cerebral palsy. Archives of physical medicine and rehabilitation, v. 92, n. 5 , p. 774-779, 2011.

KWON, J. Y.; CHANG, H. J.; YI, S. H.; LEE, J. Y.; SHIN, H. Y.; KIM, Y. H. Effect of hippotherapy on gross motor function in children with cerebral palsy: a randomized controlled trial. The Journal of Alternative and Complementary Medicine., v. 21, n. 1, p. 15-21, 2015.

LEE, C. W.; KIM, S. G.; NA, S. S. The effects of hippotherapy and a horse riding simulator on the balance of children with cerebral palsy. Journal of physical therapy science., v. 26, n. 3, p. 423-5, 2014.

LEE, N.; PARK, S.; KIM, J. Effects of hippotherapy on brain function, BDNF level, and physical fitness in children with ADHD. Journal of exercise nutrition \& biochemistry., v. 19, n. 2, p. 115-21, 2015.

LERMONTOV, T. A psicomotricidade na equoterapia. Aparecida: Idéias e Letras, 2004.

LIPORONI, G. F.; OLIVEIRA, A. P. R. Equoterapia como tratamento alternativo para pacientes com seqüelas neurológicas. Investigação, v. 5, n. 1, p. 21-29, 2010.

LIPORONI, G.; OLIVEIRA, A. P. R. Equoterapia como tratamento alternativo para pacientes com seqüelas neurológicas. Investigação., v. 5, n. 1, p. 21-9, 2010.

LONG, S. A. Hippotherapy as a Tool for Improving Motor Skills, Postural Stability, and Self Confidence in Cerebral Palsy and Multiple Sclerosis. Sound Neuroscience: An Undergraduate Neuroscience Journal., v. 1, n. 1, p. 19-40, 2014.

MEDEIROS, M.; DIAS, E.. Equoterapia: noções elementares e aspectos neurocientíficos. Rio de Janeiro: Revinter, 2008. 
MORAES, A. G.; SILVA, M.; COPETTI, F.; ABREU, A. C.; DAVID, A. C. Equoterapia no controle postural e equilíbrio em indivíduos com paralisia cerebral: revisão sistemática. Rev Neurocienc., v. 23, n. 4, p. 546-54, 2015.

SCHELBAUER, C. R.; PEREIRA, P. A. Os efeitos da equoterapia como recurso terapêutico associado com a psicomotricidade em pacientes portadores de síndrome de down. Saúde e meio ambiente: revista interdisciplinar, v. 1, n. 1, p. 117-130, 2012.

SILKWOOD-SHERER, D. J.; KILLIAN, C. B.; LONG, T. M.; MARTIN, K. S. Hippotherapy-an intervention to habilitate balance deficits in children with movement disorders: a clinical trial. Physical Therapy,v. 92, n. 5, p. 707-717, 2012.

SILVA, C. H.; GRUBITS, S. Discussão sobre o efeito positivo da equoterapia em crianças cegas. Psic: Revista da Vetor Editora, v. 5, n. 2, p. 6-13, 2004.

SMÍSKOVÁ, S. Principle and conditions of inclusion the hippotherapy in the treatment of neuromuscular disorders. Clinical Neurophysiology., v. 125, n. 5, p.36-7, 2014.

SMÍSKOVÁ, S. Principle and conditions of inclusion the hippotherapy in the treatment of neuromuscular disorders. Clinical Neurophysiology, v. 125, n. 5, p. 36-39, 2014.

SOUZA, M. B.; SILVA, P. L. N. Equoterapia no tratamento do transtorno do espectro Autista: a percepção dos técnicos. Revista Ciência e Conhecimento., v. 9, n. 1, p. 4-22, 2015.

THOMPSON, F.; KETCHAM, C. J.; HALL, E. E. Hippotherapy in children with developmental delays: Physical function and psychological benefits. Advances in Physical Education., v. 4, n. 2, p. 1-10, 2014.

TORQUATO, J. A.; LANÇA, A. F.; PEREIRA, D.; CARVALHO, F. G.; SILVA, R. D A aquisição da motricidade em crianças portadoras de Síndrome de Down que realizam fisioterapia ou praticam equoterapia. Fisioterapia em Movimento, v. 26, n. 3, p. 515-524, 2013.

TORQUATO, J. A.; LANÇA. A. F.; PEREIRA, D.; CARVALHO, F. G.; SILVA, R. D. A aquisição da motricidade em crianças portadoras de Síndrome de Down que realizam fisioterapia ou praticam equoterapia. Fisioterapia em Movimento, v. 26, n. 3, p. 515-24, 2013. 